\title{
The precuneus may encode irrationality in human gambling
}

\author{
P. Sacré, M. S. D. Kerr, S. Subramanian, K. Kahn, \\ J. Gonzalez-Martinez, M. A. Johnson, S. V. Sarma, and J. T. Gale
}

\begin{abstract}
Humans often make irrational decisions, especially psychiatric patients who have dysfunctional cognitive and emotional circuitry. Understanding the neural basis of decisionmaking is therefore essential towards patient management, yet current studies suffer from several limitations. Functional magnetic resonance imaging (fMRI) studies in humans have dominated decision-making neuroscience, but have poor temporal resolution and the blood oxygenation level-dependent signal is only a proxy for neural activity. On the other hand, lesion studies in humans used to infer functionality in decisionmaking lack characterization of neural activity altogether. Using a combination of local field potential recordings in human subjects performing a financial decision-making task, spectral analyses, and non-parametric cluster statistics, we analyzed the activity in the precuneus. In nine subjects, the neural activity modulated significantly between rational and irrational trials in the precuneus $(p<0.001)$. In particular, high-frequency activity $(70-100 \mathrm{~Hz})$ increased when irrational decisions were made. Although preliminary, these results suggest suppression of gamma rhythms via electrical stimulation in the precuneus as a therapeutic intervention for pathological decision-making.
\end{abstract}

\section{INTRODUCTION}

Decision-making links cognition to behavior and is a key driver of human personality, fundamental for survival, and essential for our ability to learn and adapt. It has been wellestablished that humans often make irrational decisions [1]. Thus, psychiatric patients who have dysfunctional cognitive circuitry, frequently have alterations in decision-making that are poorly understood.

Understanding the neural basis of decision-making is therefore essential toward patient management. However, access to the human brain has been limited to a few case studies wherein subjects have lesions in the key decisionmaking structures such as the orbital frontal cortex [2]-[4], or wherein functional magnetic resonance imaging (fMRI) is used to measure neural activity in several healthy subjects during decision-making [5]. Both of these approaches have limitations. Lesions don't provide actual neural data to ascertain a specific brain regions's role during behavior. Rather, the region's function is inferred by absence of behaviors from lesioned subjects when compared to healthy subjects. On the other hand, fMRI provides a correlate of neural

P. Sacré and S. V. Sarma are with the Institute for Computational Medicine and the Department of Biomedical Engineering, The Johns Hopkins University, Baltimore, MD (p. sacre@ jhu. edu, sree@ jhu . edu). M. S. D. Kerr is with the Department of Engineering, University of Cambridge, UK. S. Subramanian is with the Department of Clinical Neurosciences, University of Cambridge, UK. K. Kahn is with Insight Data Science, Palo Alto, CA. J. Gonzalez-Martinez, M. A. Johnson and J. T. Gale are with the Institute for Computational Medicine and the Department of Neurosciences, Cleveland Clinic, Cleveland, OH (galej@ccf.org). activity (metabolic activity) but suffers from poor temporal resolution. fMRI resolution is on the order of multiple seconds, while decisions are often made on the order of tenths of a second.

Here, we took advantage of a technique called stereoelectroencephalography (SEEG) that allowed us to record high temporal resolution electrophysiological data (electrical activity at the millisecond scale) directly from deep and peripheral brain regions in human subjects while they performed a gambling-based decision task. These subjects are implanted with electrodes for clinical purposes, and each contact in the brain generates a local field potential signal.

For our gambling task, nine subjects played a game of high card where they won virtual money if their card was higher than the computer's card. On each trial, subjects had to decide to bet "high" (\$20) or "low" (\$5) on their card being higher than the hidden computer's card. Eighty percent of the trials lead to clear rational decisions where the expected reward for one choice is higher than another. On twenty percent of the trials, however, there is no clear rational decision since the expected reward is equal for both choices. Here, we focussed on scenarios where subjects made choices that would minimize their expected reward even in scenarios where the risk of losing was low. We denote these decisions as "irrational". The goal of the study is to assess the role of different brain regions in driving these irrational decisions.

To identify neural correlates that drive irrational behavior, we computed spectrograms for each brain region (i.e., electrode channel) and each patient across all trials when irrational decisions were made and compared them to spectrograms when rational decisions were made. Specifically, we examined spectral content before, during, and after the player's card was shown and implemented a non-parametric cluster statistic to test whether the spectral activity differed between rational and irrational decisions in the given brain region. The non-parametric cluster test generates a cluster defined by a set of adjacent time-frequency windows that gives rise to the smallest $p$-value. If this $p$-value is smaller than 0.05 , then the brain region (defined by location of the channel) was designated as encoding information about (ir)rationality.

We found that upon examining several brain regions in cognitive, limbic and hippocampal networks, the precuneus encoded information that separated rational decisions from irrational decisions. Specifically, when high-frequency activity (HFA), defined as $70-100 \mathrm{~Hz}$, was prevalent in this region, then the player was more likely to make an irrational 
TABLE I

THIS TABLE PROVIDES CLINICALLY RELEVANT INFORMATION ON EACH SUBJECT: THE GENDER, THE AGE IN YEARS, AND THE DURATION OF EPILEPSY (“DUR.”) IN YEARS. THIS TABLE ALSO PROVIDES THE NUMBER OF RATIONAL AND IRRATIONAL TRIALS.

$\begin{array}{llrrrr}\text { ID } & \text { Gender } & \begin{array}{c}\text { Age } \\ \text { [yr.] }\end{array} & \begin{array}{r}\text { Dur. } \\ \text { [yr.] }\end{array} & \text { Rat. } & \text { Irr. } \\ 1 & \text { male } & 26 & 3 & 142 & 1 \\ 2 & \text { female } & 41 & 38 & 122 & 1 \\ 3 & \text { female } & 55 & 52 & 41 & 12 \\ 4 & \text { female } & 31 & 13 & 98 & 12 \\ 5 & \text { female } & 60 & 8 & 138 & 1 \\ 6 & \text { female } & 36 & 36 & 120 & 12 \\ 7 & \text { female } & 23 & 5 & 87 & 22 \\ 8 & \text { female } & 32 & 13 & 120 & 1 \\ 9 & \text { male } & 28 & 11 & 144 & 4\end{array}$

decision after he/she sees his card. The precuneus has been implicated in risk-related decision making [6], but no study has had access to electrical activity from this region at a millisecond time-scale. Hence, these findings are the first to show evidence of temporal dynamics of rhythms at a fine resolution when decisions are made. Possible therapies may include electrical stimulation to suppress such HFA to avoid irrationality.

\section{Methods}

\section{A. Subjects}

At the Cleveland Clinic, patients with medically intractable epilepsy routinely undergo SEEG recordings in order to localize the seizure focus. In this study, aside from the behavioral experiments, no alterations were made to the patient's clinical care, including the placement of the electrodes [7]. Subjects enrolled voluntarily and gave informed consent under criterion approved by the Cleveland Clinic Institutional Review Board. A total of nine subjects volunteered to perform the task. Details on these recordings and eventual annotated seizure focus of these nine patients are noted in Table I.

Subjects were implanted with 8 to 13 depth electrodes. Implantation was performed using robot-assisted surgery along with co-registered functional MRIs and angiograms to ensure safe implantation [8]. Once inserted, SEEG electrophysiological data were acquired using a Nihon Kohden 1200 EEG diagnostic and monitoring system (Nihon Kohden America, USA) at a sampling rate of $1 \mathrm{kHz}$ or $2 \mathrm{kHz}$. Behavioral event data were simultaneously acquired through the MonkeyLogic MATLAB $^{\circledR}$ toolbox [9].

\section{B. Stereoelectroencephalography}

The innovative approach using SEEG methodology relies on its capability in accessing large-scale networks, providing precise human brain data, from cortical to subcortical areas, in a three-dimensional fashion. In routine placement of depth electrodes, burr-holes that are each $15 \mathrm{~mm}$ in diameter are required for safe visualization of cortical vessels, and therefore only a small number of electrodes are placed. SEEG

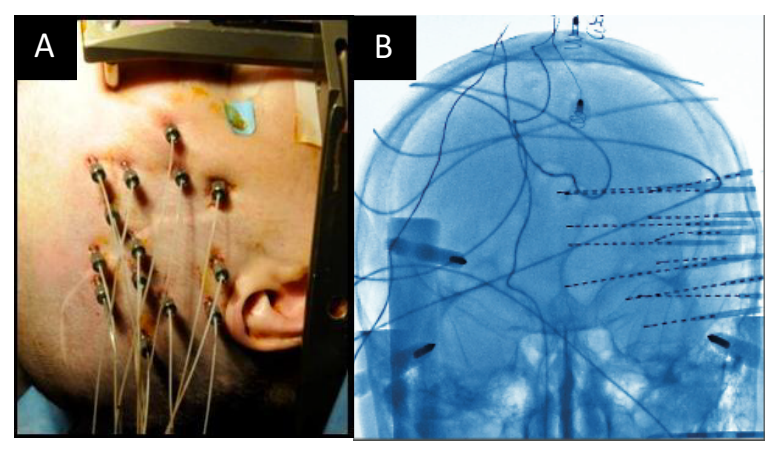

Fig. 1. Imaging fusion and placement of multiple electrodes using the SEEG method. Fig. A is a photograph showing 14 electrodes at the skin surface. Fig. B is a fluoroscopy image of an SEEG-implanted subject (coronal view with eye forward). Note the precise parallel placement, with tips terminating at the midline or dural surface.

placement, however, uses several small drill holes $(1.8 \mathrm{~mm}$ in diameter), allowing many electrodes to be inserted.

Since direct visualization of the cortical surface is not possible with small drills (Fig. 1A-B), the SEEG technique may require detailed pre-procedural vascular mapping using pre-operative imaging with magnetic resonance angiography (MRA) and cerebral angiography. Angiography is an X-ray examination of the blood vessels. The mapping procedure is performed under fluoroscopy using general anesthesia, and an expert neuro-anesthesiologist correctly titrates anesthesia to permit measurement of intracranial EEG. The number and location of implanted electrodes are pre-operatively planned based on a hypothesis, which is formulated in accordance with non-invasive pre-implantation data such as seizure semiology, ictal and inter-ictal scalp EEG, MRI images, PET and ictal single-photon emission computed tomography (SPECT) scans. Thus, the implantation strategy has the goal of accepting or rejecting the pre-implantation hypothesis of the location of the epileptogenic zone (EZ).

SEEG provides a complete coverage of the brain, from lateral, intermediate and/or deep structures in a threedimensional arrangement recorded over hundreds of channels. Using strict techniques, this procedure is safe and minimally invasive [10], [11].

\section{Gambling task}

Subjects performed the gambling task in their Epilepsy Monitoring Unit room. The task was displayed via a computer screen and the subject interacted with the task using an InMotion2 robotic manipulandum (Interactive Motion Technologies, USA). The manipulandum is controlled by the subject's hand and allows for 2D planar motion, which translated directly to the position of a cursor on screen.

The gambling task (Fig. 2A) is based on a simple game of high card where subjects would win virtual money if their card beat the computer's card. Specifically, in the beginning of each trial, the subject controlled a cursor via a planar manipulandum to a fixation target. Afterwards, the subject is shown his card $(2,4,6,8$, or 10$)$ that is randomly chosen with equal distribution (subjects are given the distribution of 


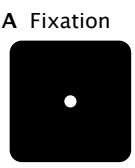

$<8 \mathrm{~s}$

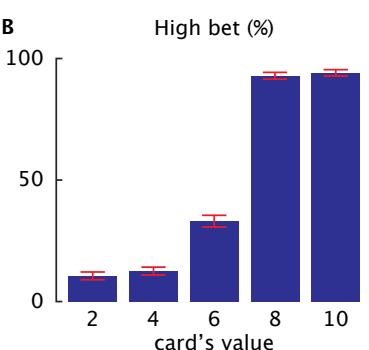

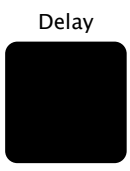

$0.35-0.6 \mathrm{~s}$

C

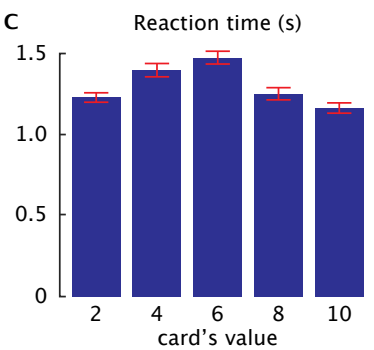

Fig. 2. Gambling task and behavioral results. (A) Timeline of the behavioral task. After fixation, subjects were shown their card. Once the bets were shown, subjects selected one of the choices and then were shown the computer's card following a delay. Feedback was provided afterwards by displaying the amount won or lost. (B) Average bet decisions across cards. Subjects predominantly bet low for 2 and 4 cards and bet high for 8 and 10 cards. There was no predominant strategy for 6 cards, which had bout $33 \%$ chance of eliciting a high bet. (C) Reaction times across cards. Subjects reacted faster for cards whose rewards had lower variability.

cards a priori). The computer's card is initially hidden. The screen then shows their two choices: a high bet $(\$ 20)$ or a low bet $(\$ 5)$. The subject has 6 seconds to select one with his cursor. Following selection, the computer's card, which follows the same distribution, is revealed. The final screen depicts the amount won or lost.

\section{Data analysis}

All electrophysiological and behavioral analyses were conducted offline using custom MATLAB ${ }^{\circledR}$ scripts.

Data for electrodes in the precuneus structure were separated into rational and irrational trials. A rational trial is defined as a trial in which the subject bets low on a 2- or 4-card, or bets high on a 8- or 10-card, thus maximizing the expected reward conditioned on their card. On the other hand, an irrational trial is defined as a trial in which the subject bets high on a 2- or 4-card, or bets low on a 8- or 10-card. Trials in which the subject receives a 6-card are ignored.

Differences in the neural responses between the task conditions during the $250 \mathrm{~ms}$ before and $750 \mathrm{~ms}$ after show card were examined by means of a non-parametric cluster statistic. Specifically, spectrograms were constructed for each trial time-locked to when the player's card is shown. Then the spectrograms for rational trials were compared to those for irrational trials. To see if spectrograms for each group were statistically significantly different, we used a nonparametric cluster-based test [12]. Clusters are defined as a set of adjacent time-frequency windows whose activity is statistically significant between trials where the subject ends up betting rationally versus irrationally.

1) Spectral analysis: We calculated the oscillatory power using multitapers from the Chronux toolbox [13]. We used three orthogonal tapers with a $300 \mathrm{~ms}$ window sliding at
$50 \mathrm{~ms}$ steps. We dropped frequencies under $10 \mathrm{~Hz}$ because of the Rayleigh criterion and analyzed upwards to $100 \mathrm{~Hz}$. Afterwards, we normalize each frequency bin's power by first taking the natural log of the power in each frequency bin, and then performing a Gaussian normalization based on the power in each frequency bin over the entire recording session.

2) Non-parametric cluster statistical test: Significant differences between the neural response data in each anatomical region are defined by a non-parametric cluster statistic run on data aggregated from trials by all relevant subjects [12].

This test leverages the dependency between adjacent timefrequency windows in order to avoid over-penalizing with multiple comparison corrections. For each time-frequency window in the spectrogram, a null distribution was created by shuffling these rational and irrational bet labels 1000 times between trials within each subject. Within each shuffle, the average difference between the newly labeled rational and irrational bet spectrograms was calculated. A $p$-value was assigned for each window by comparing the difference acquired from the true labels with the distribution of differences acquired from the shuffled labels. Clusters were formed by grouping windows with significant $p$-values $(p<0.05)$ that were adjacent in either time or frequency. The test statistic for each cluster was calculated by taking the sum of the $\log$ of the $p$-values for each window in the cluster. This prioritizes clusters that both have strong differences as well as large sizes. A null distribution of cluster statistics was created using the same process but with the 1000 spectrograms obtained from the originally shuffled labels. The observed cluster statistic was then compared against this null distribution of cluster statistics in order to obtain the final $p$-value of the test.

3) High-frequency activity: The high-frequency activity (HFA) metric captures high gamma activity and reflects previous work in SEEG [14] and other invasive recordings. HFA includes the average of all normalized log-power frequency bins between 70 and $100 \mathrm{~Hz}$ at each time point.

\section{RESULTS AND DISCUSSION}

This section summarizes the main findings of our analysis. First, we show the cluster analyses performed on precuneus neural data. Then, we show time-frequency differences of the neural responses around the show card epoch.

While multiple brain regions appear to show some response (data not shown), the two task conditions only significantly differ after show card in precuneus $(p<0.001)$. The $p$-value was computed from a non-parametric cluster statistic described in Section II.

Differences in the neural responses around the show card was examined for precuneus (Fig. 3). Spectrograms of the neural responses between rational and irrational conditions show large differences in the high frequencies after the show card. We summarize the neural activity in the time-frequency domain by averaging the responses over $70-100 \mathrm{~Hz}$. This high-frequency activity increases more for irrational trials in precuneus after the show card epoch. 

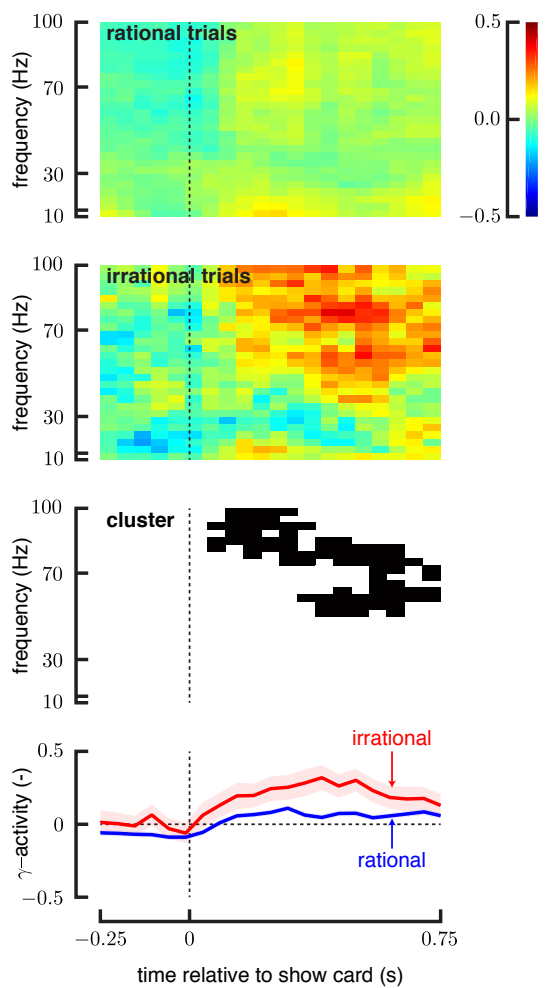

Fig. 3. Differences in the neural responses during the $250 \mathrm{~ms}$ before and $750 \mathrm{~ms}$ after the show card for precuneus. (First and second rows) Spectrograms of the neural responses show differences in the time-frequency domain between rational and irrational conditions. (Third row) Clusters emphasize the region of the time-frequency domain where the neural responses show significant differences $(p<0.05)$. (Fourth row) Highfrequency activity $(70-100 \mathrm{~Hz})$ increases after the show card for irrational trials while it doesn't modulate significantly for rational trials. Shaded error bars represent 1 standard error.

The precuneus is a structure located just above the cuneate lobe (in the parietal lobe) of the brain, near the juncture between the two hemispheres. The precuneus is interconnected to a variety of brain structures, making it integral to many systems. Consequently, the precuneus is involved in many functions generating complex human behaviors, including episodic memory, visual-spatial abilities, motor control, self perception, consciousness, and the executive and working memory.

Interestingly, the precuneus is responsible for allowing people to evaluate themselves, rating personality and physical traits. Its self-reflection allows comparisons to be made between the self and others, and informs societal behavior. A recent fMRI study suggested that the precuneus is involved in "reasoning in the social world" [15] and rationality. The authors use the definition of rationality proposed by "communicative action theory" [16], where rationality refers to interpersonal communication rather than to a knowing subject. They showed that the precuneus, among other regions, activated during communicative reasoning.

In our subject population, the neural activity in the precuneus modulated significantly between rational and irrational trials $(p<0.001)$. In particular, high-frequency activity increased after the show card for irrational trials. Such high frequency temporal dynamics cannot be observed with fMRI, yet decisions are made at such a fine temporal scale.

\section{Conclusion}

In conclusion, our findings suggest that the precuneus should be taken into account in decision-making studies for examining the role of rationality as defined by maximizing a utility function such as the expected reward. Still, the exact mechanism by which the precuneus is involved in decision making is not known and will therefore remains the focus of future work.

\section{ACKNOWLEDGMENT}

This work was supported by a National Science Foundation grant (EFRI-MC3: \# 1137237) awarded to S.V.S., J.A.G., J.B. and J.T.G.

\section{REFERENCES}

[1] M. Toda, "Emotion and decision making," Acta Psychologica, vol. 45, no. 1-3, pp. 133-155, 1980.

[2] A. Bechara, "The role of emotion in decision-making: Evidence from neurological patients with orbitofrontal damage," Brain and Cognition, vol. 55 , no. 1 , pp. 30-40, 2004.

[3] J. Kim and M. E. Ragozzino, "The involvement of the orbitofrontal cortex in learning under changing task contingencies," Neurobiology of Learning and Memory, vol. 83, no. 2, pp. 125-133, 2005.

[4] I. Bohn, C. Giertler, and W. Hauber, "Orbital prefrontal cortex and guidance of instrumental behaviour in rats under reversal conditions," Behavioural Brain Research, vol. 143, no. 1, pp. 49-56, 2003.

[5] N. K. Logothetis, "What we can do and what we cannot do with fMRI," Nature, vol. 453, pp. 869-878, June 2008.

[6] G. I. Christopoulos, P. N. Tobler, P. Bossaerts, R. J. Dolan, and W. Schultz, "Neural correlates of value, risk, and risk aversion contributing to decision making under risk," The Journal of Neuroscience, vol. 29, no. 40, pp. 12574-12583, 2009.

[7] M. A. Johnson, S. Thompson, J. Gonzalez-Martinez, H.-J. Park, J. Bulacio, I. Najm, K. Kahn, M. Kerr, S. V. Sarma, and J. T. Gale, "Performing behavioral tasks in subjects with intracranial electrodes," Journal of Visualized Experiments, no. 92, p. e51947, 2014.

[8] D. Öngür and J. Price, "The organization of networks within the orbital and medial prefrontal cortex of rats, monkeys and humans," Cerebral Cortex, vol. 10, no. 3, pp. 206-219, 2000

[9] W. F. Asaad and E. N. Eskandar, "A flexible software tool for temporally-precise behavioral control in Matlab," Journal of Neuroscience Methods, vol. 174, no. 2, pp. 245-258, 2008.

[10] J. Gonzalez-Martinez, J. Mullin, S. Vadera, J. Bulacio, G. Hughes, S. Jones, R. Enatsu, and I. Najm, "Stereotactic placement of depth electrodes in medically intractable epilepsy," Journal of Neurosurgery, vol. 120, no. 3, pp. 639-644, 2014.

[11] F. Cardinale and G. Lo Russo, "Stereo-electroencephalography safety and effectiveness: Some more reasons in favor of epilepsy surgery," Epilepsia, vol. 54, no. 8, pp. 1505-1506, 2013.

[12] E. Maris and R. Oostenveld, "Nonparametric statistical testing of EEGand MEG-data," Journal of Neuroscience Methods, vol. 164, no. 1, pp. 177-190, 2007.

[13] H. Bokil, P. Andrews, J. E. Kulkarni, S. Mehta, and P. P. Mitra, "Chronux: A platform for analyzing neural signals," Journal of Neuroscience Methods, vol. 192, no. 1, pp. 146-151, 2010.

[14] T. Ossandón, K. Jerbi, J. R. Vidal, D. J. Bayle, M.-A. Henaff, J. Jung, L. Minotti, O. Bertrand, P. Kahane, and J.-P. Lachaux, "Transient suppression of broadband gamma power in the default-mode network is correlated with task complexity and subject performance," The Journal of Neuroscience, vol. 31, no. 41, pp. 14521-14530, 2011.

[15] M. Schaefer, H.-J. Heinze, M. Rotte, and C. Denke, "Communicative versus strategic rationality: Habermas theory of communicative action and the social brain," PLOS ONE, vol. 8, p. e65111, May 2013.

[16] T. McCarthy, "Translator's introduction," in The theory of communicative action: Reason and the Rationalization of Society (J. Habermas, ed.), Beacon Press, 1984. 